\title{
TARGET SELECTION FOR THE LBTI EXOZODI KEY SCIENCE PROGRAM
}

\author{
Alycia J. Weinberger ${ }^{1}$, Geoff Bryden ${ }^{2}$, Grant M. Kennedy ${ }^{3}$, Aki Roberge ${ }^{4}$, Denis Defrère ${ }^{5}$, Philip M. Hinz ${ }^{5}$, \\ Rafael Millan-Gabet ${ }^{6}$, George Rieke ${ }^{5}$, Vanessa P. Bailey ${ }^{5}$, William C. Danchi ${ }^{4}$, Chris HanifF ${ }^{7}$, \\ Bertrand Mennesson ${ }^{2}$, Eugene Serabyn ${ }^{2}$, Andrew J. Skemer ${ }^{5}$, Karl R. Stapelfeldt ${ }^{4}$, and Mark C. Wyatt ${ }^{3}$ \\ ${ }^{1}$ Department of Terrestrial Magnetism, Carnegie Institution for Science, 5241 Broad Branch Road NW, Washington, DC 20015, USA; weinberger@dtm.ciw.edu \\ 2 Jet Propulsion Laboratory, California Institute of Technology, 4800 Oak Grove Dr, Pasadena, CA 91109, USA \\ ${ }^{3}$ Institute of Astronomy, University of Cambridge, Madingley Road, Cambridge CB3 0HA, UK \\ ${ }^{4}$ Exoplanets \& Stellar Astrophysics Laboratory, NASA Goddard Space Flight Center, Code 667, Greenbelt, MD 20771, USA \\ ${ }^{5}$ Steward Observatory, University of Arizona, 933 North Cherry Lane, Tucson, AZ 85721, USA \\ ${ }^{6}$ NASA Exoplanet Science Institute, California Institute of Technology, Pasadena, CA 91125, USA \\ ${ }^{7}$ Cavendish Laboratory, University of Cambridge, JJ Thomson Avenue, Cambridge CB3 0HE, UK \\ Received 2014 October 2; accepted 2014 December 12; published 2015 January 27
}

\begin{abstract}
The Hunt for Observable Signatures of Terrestrial planetary Systems (HOSTS) on the Large Binocular Telescope Interferometer will survey nearby stars for faint emission arising from $\sim 300 \mathrm{~K}$ dust (exozodiacal dust), and aims to determine the exozodiacal dust luminosity function. HOSTS results will enable planning for future space telescopes aimed at direct spectroscopy of habitable zone terrestrial planets, as well as greater understanding of the evolution of exozodiacal disks and planetary systems. We lay out here the considerations that lead to the final HOSTS target list. Our target selection strategy maximizes the ability of the survey to constrain the exozodi luminosity function by selecting a combination of stars selected for suitability as targets of future missions and as sensitive exozodi probes. With a survey of approximately 50 stars, we show that HOSTS can enable an understanding of the statistical distribution of warm dust around various types of stars and is robust to the effects of varying levels of survey sensitivity induced by weather conditions.
\end{abstract}

Key words: circumstellar matter - surveys - techniques: interferometric

\section{INTRODUCTION}

The Hunt for Observable Signatures of Terrestrial planetary Systems (HOSTS) on the Large Binocular Telescope Interferometer (LBTI) will survey nearby stars for faint exozodiacal dust (exozodi). This warm circumstellar dust, such as that found in the vicinity of Earth, is generated in asteroidal collisions and cometary breakups. We define exozodiacal dust as sitting in the habitable zone, that is, $\sim 1 \mathrm{AU}$ from a Solar-type star, and therefore as having a temperature comparable to the Earth, i.e., $\sim 278 \mathrm{~K}$.

The goal of the LBTI HOSTS survey is to provide information on exozodi needed to develop a future space telescope aimed at direct detection of habitable zone terrestrial planets (aka. exoEarths). The habitable zone is defined by where a terrestrial planet can have long-term surface water, but its exact boundaries depend on planetary properties. Nevertheless, surface temperatures near $300 \mathrm{~K}$ imply that Earth-mass exoplanets need insolations comparable to that of Earth up to 1.2 times greater than Earth's (e.g., Leconte et al. 2013; Kopparapu et al. 2013). There is no single agreed upon definition of exozodi in the literature (Roberge et al. 2012). The HOSTS team has adopted a definition that scales the surface density of the Sun's Zodiacal disk at the Earth equivalent insolation distance (EEID). Thus the surface density profile expands with stellar luminosity, and allows the "exozodi" level to be compared across stars of different types. See the companion paper Kennedy et al. (2014) for a full discussion of our adopted model. This reference model includes dust interior to the habitable zone all the way in to the sublimation radius, so this model may test how close-in dust such as that detected in near-infrared interferometric surveys (Absil et al. 2013; Ertel et al. 2014) is related to habitable zone dust.
The typical exozodi detection from space-based photometry and spectrophotometry, primarily with the IRS instrument on the Spitzer Space Telescope, is $\sim 1000$ times the solar system's

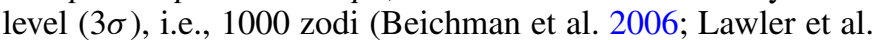
2009; Chen et al. 2014). The best limits from the groundbased Keck interferometer are 500 zodi $(3 \sigma$; Millan-Gabet et al. 2011; Mennesson et al. 2014). Interferometric searches for dust in the near-infrared can find dust interior to the habitable zone, at temperatures $\gtrsim 500 \mathrm{~K}$ (Absil et al. 2013; Ertel et al. 2014 ) or that comes from scattered light, and far-infrared and submillimeter telescopes can find dust much cooler than exozodi, at temperatures $<100 \mathrm{~K}$ (e.g., Eiroa et al. 2013). LBTIHOSTS will be the first survey capable of measuring exozodi known to be at habitable zone temperatures and at the $10-20$ zodi level $(3 \sigma)$.

Exozodi of this brightness would be the major source of astrophysical noise for a future space telescope aimed at direct imaging and spectroscopy of habitable zone terrestrial planets. For example, more than about 4 zodis would cause the integration time for coronagraphic imaging of an Earth-like planet in the habitable zone of a G2V star at $10 \mathrm{pc}$ to exceed one day, using a $4 \mathrm{~m}$ telescope and the other baseline astrophysical and mission parameters given in Stark et al. (2014). A larger telescope can tolerate larger zodi levels for the same integration time.

Detections of warm dust will also reveal new information about planetary system architectures and evolution. Asteroid belts undergoing steady state collisions should grind themselves down in much less time than the Gyr ages of nearby stars. So, warm debris disks around old stars may signal late cometary influxes or stochastic collisional events (e.g., Wyatt et al. 2007; Gáspár et al. 2013). While 20\% of nearby stars have cold, i.e., $<150 \mathrm{~K}$, dust (Eiroa et al. 2013) and $\sim 15 \%$ have 
hot, i.e., $>500 \mathrm{~K}$, dust (Ertel et al. 2014), there is presently no demonstrated connection between the two. To understand the evolution of planetary systems, we seek to measure the luminosity function of exozodi with age and stellar mass and determine whether the presence of cold outer disks correlates with warm inner exozodi.

LBTI is a nulling interferometer, designed to use the $8.4 \mathrm{~m}$ apertures of the LBT fixed in a common mount at a $14.4 \mathrm{~m}$ separation, for the detection of emission from warm dust around nearby stars. LBTI works in the thermal infrared, employing dual adaptive secondaries to correct atmospheric seeing, and providing low thermal background and high Strehl images to the science camera NOMIC (Hinz et al. 2008, 2012). Closed loop phase tracking in the near infrared is used to stabilize the destructive interference of the star in the $N^{\prime}$ band $(9.8-12.4 \mu \mathrm{m})$ and detect flux from the resolved dust disk Defrère et al. (2014). The separation of the LBT mirrors at a working wavelength of $11 \mu \mathrm{m}$ produces a first transmission peak centered at 79 mas (1 $\mathrm{AU}$ at $13 \mathrm{pc}$ ) and an inner working angle (half transmission) of 39 mas (1 AU at $25 \mathrm{pc}$ ).

Together, observations of thermal emission from disks with LBTI and images with space-based optical coronagraphs capable of probing the same angular scales in scattered light will measure the albedo of dust grains. Albedo is one of the few available constraints on dust composition and thereby parent body composition for debris disks. Scattered light images of dust in the habitable zones of several nearby stars may be possible with a coronagraph on the WFIRST-AFTA mission (Spergel et al. 2013).

\section{TARGET LIST ASSEMBLY AND EXCLUSIONS}

\subsection{Target Selection Goals}

Target selection for HOSTS is a balance between including stars that are expected targets of a future exoEarth mission and including stars of various types to enable the best understanding of the statistical distribution of exozodi over a range of parameters. The two approaches are complementary and together enable investigations of habitable zone dust production across a range of host stellar types.

The mission-driven approach concentrates on F-, G-, and K-type stars that are the best targets for future direct observations of exoEarths, thereby providing "ground truth" dust observations. The sensitivity sweet spot for an optical planet imager lies with $\mathrm{G}$ and $\mathrm{K}$ stars because (1) the planet-to-star contrast ratio is inversely proportional to stellar luminosity and (2) the orbital radius of the habitable zone increases as $\sqrt{L_{*}} .8$ As a result, M-type stars have favorable planet-to-star contrast ratios but habitable zones close to the stars, whereas A-type stars have poor contrast ratios and habitable zones further from the stars.

Not every potential target of a future exoEarth mission can be observed with LBTI; for one thing, many lie in the southern hemisphere and are not observable from LBT on Mount Graham, AZ. Furthermore, some stars bright enough at visual

\footnotetext{
8 The EEID, i.e., where a planet receives the same incident flux as Earth, defines the habitable zone (see Section 3). Since the flux at the EEID is a constant, a $1 R_{\oplus}$ planet there always has the same absolute magnitude independent of host star luminosity. However, the absolute magnitude of stars decreases toward earlier spectral type stars, thus increasing the star-to-planet flux ratio. The radial temperature dependence of a blackbody emitter in a stellar radiation field can be calculated by equating the incident flux $\left(L_{*} / 4 \pi\right)$ with the emergent flux $\left(4 \sigma r_{\mathrm{EEID}}^{2} T_{\mathrm{HZ}}^{4}\right)$. Thus, for a fixed temperature, as in a habitable zone, the radius at which a blackbody reaches that temperature is proportional to $\sqrt{L}$.
}

wavelengths and therefore accessible to an exoEarth mission would be too faint for LBTI to achieve good sensitivity in the limited total observing time. Our goal is to design a survey that can fully inform target selection for a future exoEarth mission; survey results will have to be modeled and then extrapolated to lower dust levels. Therefore, there must be observational extensions to the mission-driven sample that will inform models of dust evolution and aid extrapolation.

The second approach, a LBTI sensitivity-driven approach, selects targets based only on the expected LBTI exozodi sensitivities, without consideration of exoEarth mission constraints. This would naturally select more early-type stars (A stars and early F-type stars) because they are brighter, have habitable zones at large separations, and higher $F_{\text {disk }} / F_{*}$ in the $N$ band (see Kennedy et al. 2014, for details). Therefore, the results of this type of survey would have to be extrapolated to later spectral type targets using planet formation theory.

The brightest nearby late-F- to K-type stars can satisfy both the mission and sensitivity-driven selection criteria, and we give a description of these in Section 3, we show that there are 25-48 such stars, depending on LBTI sensitivity. We anticipate that HOSTS will survey $\sim 50$ stars, given the amount of observing time allocated on LBTI, so the target selection approach followed will determine the rest of the observed stars.

We lay out here the considerations that lead to the final HOSTS target list. We discuss how to balance mission-driven and sensitivity considerations to maximize scientific return from the HOSTS project. By presenting our target list in this early paper, we also hope to encourage intensive study of these stars with other techniques that will eventually enhance our ability to understand the evolution of circumstellar dust with time.

\subsection{Target Selection Constraints}

We started with a list of all bright, northern main-sequence stars of spectral types A through $\mathrm{M}$ observable from LBT (declination $>-30^{\circ}$ ) by using two catalogs: the Unbiased Nearby Stars (UNS) sample assembled for cold debris disks studies (Phillips et al. 2010) and the Hipparcos 30 pc sample assembled for exoEarth mission planning (Turnbull et al. 2012). UNS is complete to about $16 \mathrm{pc}$ for K-type stars and about $45 \mathrm{pc}$ for A type.

Binary stars were excluded based on both technical and scientific criteria. There are two technical reasons to exclude binary stars: (1) to ensure that the adaptive optics system can easily lock on the target of interest and (2) to ensure that flux from the companion does not contaminate the area being searched for exozodi emission. We therefore excluded binary stars with separations $<1^{\prime \prime} .5$. Some stars are known to be spectroscopic binaries (SBs) but without well-measured orbits. We excluded all such SBs because their maximum separations might fall within an angular range of $10 \mathrm{~s}$ to $100 \mathrm{~s}$ of mas and provide confusing non-null signals. The main sources of information about multiplicity were the Washington Visual Double Star Catalog (Mason et al. 2013) and the 9th Catalogue of Spectroscopic Binary Orbits (Pourbaix et al. 2009).

We further excluded stars with flux densities $<1 \mathrm{Jy}$ in the broad $N$-band $(\sim 11 \mu \mathrm{m})$ filter used for the HOSTS survey. We anticipate that the LBTI null would be degraded for fainter stars. To estimate the brightness of our targets, we fit Kurucz stellar models to available photometry at BVJHK plus WISE bands W3 $(12 \mu \mathrm{m})$ and W4 $(22 \mu \mathrm{m})$ and then used the model to predict the NOMIC flux density. 
We also only considered stars with inner habitable zone distances probed by the LBTI transmission pattern, i.e., zones larger than about 60 mas. An exozodi disk smaller than this has low transmission efficiency, i.e., it is nulled along with the star because the LBTI transmission pattern peak is at $\approx 79$ mas. A general result of our brightness cuts is that our target stars are all within $28 \mathrm{pc}$. Therefore, our angular criterion, above, excluded binaries with separations $\lesssim 50$ AU. Furthermore, studies of protoplanetary disk evolution indicate that stellar companions within $100 \mathrm{AU}$ of the primary stars cause lower disk masses and faster disk dissipation, possibly inhibiting planet formation (e.g., Osterloh \& Beckwith 1995; Jensen et al. 1996; Andrews \& Williams 2005; Harris et al. 2012). We therefore also excluded physical binaries with separations $<100$ AU. Although it would be interesting to study the effect of binary separation on habitable zone dust, we emphasized the formation of an overall sample with as few selection effects as possible and eschewed inclusion of subsamples too small to provide statistically meaningful results.

Finally, we excluded giant stars (luminosity class III), i.e., stars that appear significantly brighter than the main sequence. LBTI would probe regions around these stars that are significantly larger than the size of the habitable zones that existed when the stars resided on the main sequence and thus not directly comparable to the rest of the sample. Table 3 lists the targets excluded for binarity and location above the main sequence.

\subsection{Target List Categories}

We categorize the targets that meet the above criteria into two samples described below.

Section 3: the Sun-like sample includes targets with spectral types later than F5. These 48 stars are potential targets for a future exoEarth mission. Of these 25 have flux density $>2 \mathrm{Jy}$ in the $N$ band.

Section 4: the sensitivity-driven, i.e., early-type star, sample includes targets with spectral types between A0 and F4. These 20 stars provide additional information on the exozodi luminosity function. Of these, 15 have flux density $>2 \mathrm{Jy}$ in the $N$ band.

Together, there are 68 sources in the above categories from which the optimal HOSTS survey can be created.

\section{SUN-LIKE SAMPLE}

Our objective for this LBTI-HOSTS sub-sample is to observe stars that are probable targets for a future exoEarth mission, based on current knowledge, and stars that inform our understanding of the typical exozodi levels around similar stars. These observations will provide dust measurements (or upper limits) for specific stars. They will also supply a larger sample of solartype stars with which to model the distribution of exozodi levels for Sun-like stars. This will enable evaluation of the suitability, as exoEarth mission targets, of individual stars that could not be observed from LBTI (because, for example, they were too faint or too far south). Here, we define "Sun-like" as having spectral types later than or equal to F5. The coolest star that passed all our cuts is spectral type K8. The majority of high photometric quality targets for the Kepler mission's exoplanet search are also of spectral types mid-K to mid-F (4500-7000 K; Christiansen et al. 2012).

The great technical challenge for direct exoEarth observations is to suppress the central starlight tremendously yet allow light from extremely faint planets to be detected at small angular separations. Therefore, the best systems to search for exoEarths

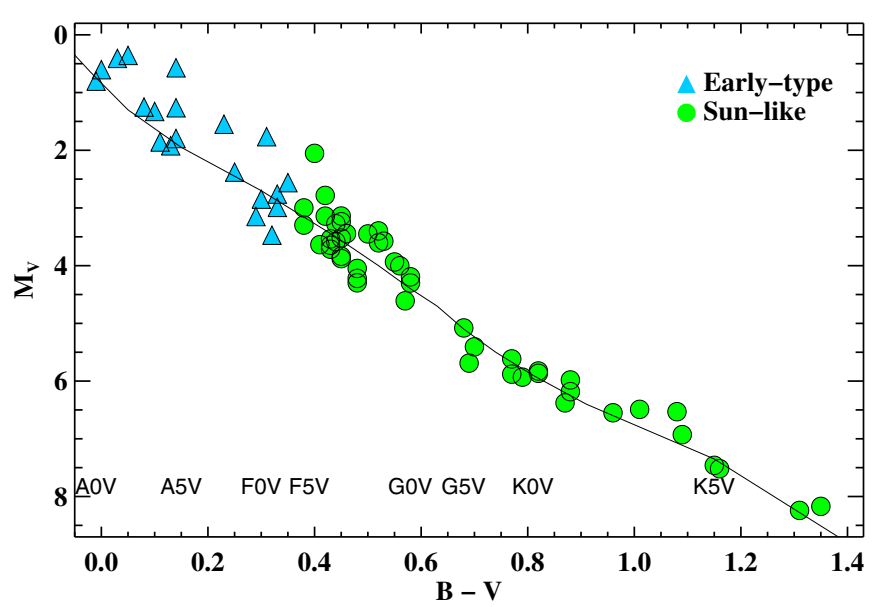

Figure 1. Color-magnitude (absolute $V$ magnitude vs. $B-V$ color) plot of the complete sample. Sun-like stars, defined as spectral type F5 and later, are shown with green filled circles. Early type stars, defined as spectral types F4 and earlier, are show with blue filled triangles. The black line shows the MK main sequence as given in Drilling \& Landolt (2000).

are those with widely separated habitable zones (HZs) and with high planet-to-star flux ratios. A full discussion of all the considerations that go into determining a star's habitable zone boundaries appears in Kopparapu et al. (2013). However, to first order, the location of a star's HZ is set by how much light would hit an Earth-twin planet. Therefore, the EEID approximately scales in the following way:

$$
r_{\mathrm{EEID}} \approx r_{\oplus} \times\left(L_{\star} / L_{\odot}\right)^{1 / 2}
$$

where $L$ is the bolometric luminosity and $r_{\oplus}$ is the Earth-Sun distance.

Following Turnbull et al. (2012), the planet-to-star reflected flux ratio at visible wavelengths for an Earth-twin planet is approximately

$$
\left(F_{p} / F_{\star}\right)_{\mathrm{HZ}} \approx\left(1.2 \times 10^{-10}\right) /\left(L_{\star} / L_{\odot}\right)
$$

So as the stellar luminosity increases, the HZ moves outward, increasing the separation of an exoEarth from its host star (good). However, simultaneously the planet-to-star flux ratio decreases, resulting in longer exposure times to reach a given detection limit (bad).

These two competing effects largely dictate which stars are the best for direct observations of exoEarths. The current consensus is that starlight suppression technologies working at optical wavelengths (e.g., internal coronagraphs) are the most advanced (Greene et al. 2013). For these mission concepts, the best targets are nearby stars of mid-F, G, and K spectral types. In general, for a given optical coronagraphic telescope aperture, the less exozodi noise contribution that a star system has, the earlier the spectral type of star systems that can be searched with high completeness. An interferometric mission, such as an array of $4 \times 4 \mathrm{~m}$ free-flying mid-infrared telescopes, provides somewhat different completeness as a function of stellar luminosity. For the HOSTS survey, we make no assumptions about exoEarth detection technology. If we keep the ratio of F:G:K stars fixed, the best targets for an interferometric telescope agree well with the coronagraphic telescope list (Defrère et al. 2010).

We found 48 stars that met all of our selection criteria and some of their basic properties are listed in Table 1 and shown in Figures 1 and 2. Our current working knowledge of the 
Table 1

Stars in the Sun-like Star Sample

\begin{tabular}{|c|c|c|c|c|c|c|c|c|c|}
\hline HD & Name & R.A. & Decl. & $\begin{array}{l}\text { Distance } \\
\quad(\mathrm{pc})\end{array}$ & $\begin{array}{l}\text { Spectral } \\
\text { Type }\end{array}$ & EEID & $\begin{array}{c}F_{v}(N \text { band }) \\
(\mathrm{Jy})\end{array}$ & $\begin{array}{c}\text { Hot/Warm } \\
\text { Excess }\end{array}$ & $\begin{array}{c}\text { Cold } \\
\text { Excess }\end{array}$ \\
\hline 693 & $6 \mathrm{Cet}$ & $00: 11: 15.86$ & $-15: 28: 04.7$ & 18.7 & F8V & 0.095 & 1.15 & & $\mathrm{n}(\mathrm{E} 13)$ \\
\hline 4628 & & $00: 48: 22.98$ & $+05: 16: 50.2$ & 7.5 & $\mathrm{~K} 2.5 \mathrm{~V}$ & 0.072 & 1.30 & & n (T08) \\
\hline 9826 & $v$ And & $01: 36: 47.84$ & $+41: 24: 19.6$ & 3.5 & F9V & 0.136 & 2.36 & n (A13) & $\mathrm{n}(\mathrm{B} 06, \mathrm{E} 13)$ \\
\hline 10476 & 107 Psc & $01: 42: 29.76$ & $+20: 16: 06.6$ & 7.5 & $\mathrm{~K} 1 \mathrm{~V}$ & 0.090 & 2.02 & n (MG11) & n (T08) \\
\hline 10700 & tau Cet & 01:44:04.08 & $-15: 56: 14.9$ & 3.6 & G8.5V & 0.182 & 5.42 & y (A13) & y (G04) \\
\hline 10780 & GJ 75 & $01: 47: 44.83$ & $+63: 51: 09.0$ & 10.1 & K0V & 0.072 & 1.12 & & n (L09) \\
\hline 16160 & GJ 105 & 02:36:04.89 & $+06: 53: 12.7$ & 7.2 & $\mathrm{~K} 3 \mathrm{~V}$ & 0.073 & 1.53 & & n (T08) \\
\hline 16895 & 13 Per & 02:44:11.99 & $+49: 13: 42.4$ & 11.1 & F7V & 0.138 & 2.43 & n (A13) & n (Be06) \\
\hline 17206 & tau01 Eri & $02: 45: 06.19$ & $-18: 34: 21.2$ & 14.2 & F75 & 0.115 & 1.69 & & n (T08) \\
\hline 19373 & iot Per & 03:09:04.02 & $+49: 36: 47.8$ & 10.5 & F9.5V & 0.141 & 2.85 & n (MG11) & n (T08) \\
\hline 22049 & eps Eri & $03: 32: 55.84$ & $-09: 27: 29.7$ & 3.2 & $\mathrm{~K} 2 \mathrm{~V}$ & 0.172 & 7.39 & n (A13) & y (B09) \\
\hline 22484 & LHS 1569 & $03: 36: 52.38$ & $+00: 24: 06.0$ & 14.0 & F8V & 0.127 & 2.35 & y (A13) & y (T08) \\
\hline 23754 & tau06 Eri & $03: 46: 50.89$ & $-23: 14: 59.0$ & 17.6 & F5IV-V & 0.128 & 2.10 & & $\mathrm{n}(\mathrm{G} 13)$ \\
\hline 26965 & omi Eri & $04: 15: 16.32$ & $-07: 39: 10.3$ & 5.0 & K0.5V & 0.128 & 3.51 & & n (L02) \\
\hline 30652 & 1 Ori & $04: 49: 50.41$ & $+06: 57: 40.6$ & 8.1 & F6V & 0.205 & 4.76 & n (A13) & n (T08) \\
\hline 32147 & & 05:00:49.00 & $-05: 45: 13.2$ & 8.7 & $\mathrm{~K} 3 \mathrm{~V}$ & 0.059 & 1.00 & & n (L09) \\
\hline 34411 & lam Aur & 05:19:08.47 & $+40: 05: 56.6$ & 12.6 & G1.5IV & 0.105 & 1.80 & n (MG11) & n (T08) \\
\hline 35296 & V1119 Tau & $05: 24: 25.46$ & $+17: 23: 00.7$ & 14.4 & F8V & 0.090 & 1.03 & & n (T08) \\
\hline 38393 & gam Lep & $05: 44: 27.79$ & $-22: 26: 54.2$ & 8.9 & F6V & 0.175 & 4.40 & n (MG11) & n (Be06) \\
\hline 48737 & ksi Gem & $06: 45: 17.36$ & $12: 53: 44.13$ & 18.0 & F5IV & 0.196 & 4.34 & $\mathrm{n}(\mathrm{A} 13)$ & $\mathrm{n}(\mathrm{K} 13)$ \\
\hline 78154 & sig02 Uma A & $09: 10: 23.54$ & $+67: 08: 02.4$ & 20.4 & F6IV & 0.099 & 1.24 & & n (G13) \\
\hline 84117 & GJ 364 & $09: 42: 14.42$ & $-23: 54: 56.0$ & 15.0 & F8V & 0.093 & 1.11 & & n (E13) \\
\hline 88230 & NSV 4765 & $10: 11: 22.14$ & $+49: 27: 15.3$ & 4.9 & K8V & 0.065 & 1.91 & n (MG11) & n (T08) \\
\hline 89449 & 40 Leo & 10:19:44.17 & $+19: 28: 15.3$ & 21.4 & F6IV & 0.098 & 1.10 & & n (G13) \\
\hline 90839 & 36 Uma & $10: 30: 37.58$ & $+55: 58: 49.9$ & 12.8 & F8V & 0.099 & 1.25 & & n (T08) \\
\hline 95128 & 47 Uma & $10: 59: 27.97$ & $+40: 25: 48.9$ & 14.1 & G1V & 0.091 & 1.35 & n (MG11) & n (T08) \\
\hline 101501 & $61 \mathrm{Uma}$ & 11:41:03.02 & $+34: 12: 05.9$ & 9.6 & G8V & 0.081 & 1.24 & & n (G03) \\
\hline 102870 & bet Vir & $11: 50: 41.72$ & $+01: 45: 53.0$ & 10.9 & F9V & 0.173 & 4.30 & n (A13) & n (T08) \\
\hline 115617 & $61 \mathrm{Vir}$ & $13: 18: 24.31$ & $-18: 18: 40.3$ & 8.6 & G7V & 0.108 & 2.20 & n (L09, E14) & y (L09) \\
\hline 120136 & tau Boo & $13: 47: 15.74$ & $+17: 27: 24.8$ & 15.6 & F6IV & 0.114 & 1.67 & n (E14) & n (B09) \\
\hline 126660 & tet Boo & $14: 25: 11.8$ & $+51: 51: 02.7$ & 14.5 & F7V & 0.147 & 3.12 & & n (T08) \\
\hline 131977 & KX Lib & $14: 57: 28.00$ & $-21: 24: 55.7$ & 5.8 & $\mathrm{~K} 4 \mathrm{~V}$ & 0.076 & 1.95 & n (L09) & n (Be06) \\
\hline 141004 & lam Ser & $15: 46: 26.61$ & $+07: 21: 11.0$ & 12.1 & G0IV-V & 0.121 & 2.40 & n (A13) & n (K10) \\
\hline 142373 & LHS 3127 & $15: 52: 40.54$ & $+42: 27: 05.5$ & 15.9 & F8Ve & 0.111 & 2.03 & n (A13) & n (T08) \\
\hline 142860 & gam Ser & $15: 56: 27.18$ & $+15: 39: 41.8$ & 11.2 & F6IV & 0.151 & 2.93 & n (A13) & n (T08) \\
\hline 149661 & V2133 Oph & $16: 36: 21.45$ & $-02: 19: 28.5$ & 9.8 & $\mathrm{~K} 2 \mathrm{~V}$ & 0.068 & 1.00 & n (E14) & n (T08) \\
\hline 156026 & V2215 Oph & $17: 16: 13.36$ & $-26: 32: 46.1$ & 6.0 & $\mathrm{~K} 5 \mathrm{~V}$ & 0.064 & 1.64 & & n (Be06) \\
\hline 157214 & w Her & $17: 20: 39.30$ & $+32: 28: 21.2$ & 14.3 & G0V & 0.079 & 1.01 & & n (T08) \\
\hline 160915 & $58 \mathrm{Oph}$ & $17: 43: 25.79$ & $-21: 40: 59.5$ & 17.6 & F5V & 0.096 & 1.18 & n (E14) & n (E14) \\
\hline 173667 & $110 \mathrm{Her}$ & $18: 45: 39.72$ & $+20: 32: 46.7$ & 19.2 & F6V & 0.131 & 2.18 & y (A13) & n (T08) \\
\hline 185144 & sig Dra & $19: 32: 21.59$ & $+69: 39: 40.2$ & 5.8 & G9V & 0.113 & 2.72 & $\mathrm{n}(\mathrm{A} 13)$ & n (T08) \\
\hline 192310 & GJ 785 & $20: 15: 17.39$ & $-27: 01: 58.7$ & 8.9 & $\mathrm{~K} 2+\mathrm{V}$ & 0.071 & 1.25 & & n (Be06) \\
\hline 197692 & psi Cap & $20: 46: 05.73$ & $-25: 16: 15.2$ & 14.7 & F5V & 0.136 & 2.08 & n (E14) & n (L09) \\
\hline 201091 & 61 Cyg A & $21: 06: 53.95$ & $+38: 44: 58.0$ & 3.5 & $\mathrm{~K} 5 \mathrm{~V}$ & 0.106 & 4.43 & n (A13) & n (G04) \\
\hline 201092 & 61 Cyg B & $21: 06: 55.26$ & $+38: 44: 31.4$ & 3.5 & $\mathrm{~K} 7 \mathrm{~V}$ & 0.085 & 3.28 & n (A13) & n (G04) \\
\hline 215648 & ksi Peg A & $22: 46: 41.58$ & $+12: 10: 22.4$ & 16.3 & F7V & 0.132 & 2.22 & n (E14) & n (G13) \\
\hline 219134 & & $23: 13: 16.98$ & $+57: 10: 06.1$ & 6.5 & $\mathrm{~K} 3 \mathrm{~V}$ & 0.080 & 1.86 & & n (T08) \\
\hline 222368 & iot Psc & $23: 39: 57.04$ & $+05: 37: 34.6$ & 13.7 & F7V & 0.137 & 2.40 & n (MG11) & n (B06) \\
\hline
\end{tabular}

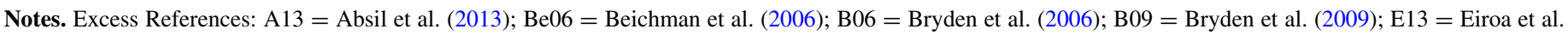

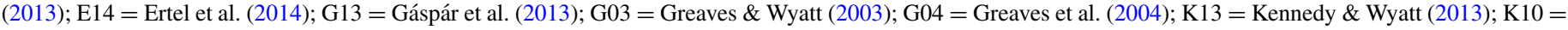
Koerner et al. (2010); L02 = Laureijs et al. (2002); L09 = Lawler et al. (2009); MG11 = Millan-Gabet et al. (2011); T08 = Trilling et al. (2008).

LBTI system is that the null quality will not depend on stellar brightness for stars brighter than $2 \mathrm{Jy}$ in the $N$ band; there are 25 such bright stars on our list. We expect that for stars fainter than $2 \mathrm{Jy}$, the degradation in the null will be a gentle function of brightness, but this remains to be tested.

The mean distance to these sample stars is $11.4 \mathrm{pc}$; the closest star is at $3.2 \mathrm{pc}$ and the most distant at $21.4 \mathrm{pc}$. The presence/ absence of a disk was not a criterion for selecting stars in the Sunlike star sample. What is known about the presence or absence of hot/warm (potentially $\mathrm{N}$-band detectable) and cold (far-IR detectable) circumstellar disks is noted in the table. Each dust survey has somewhat different limits; the reader should consult the original papers for details.

\section{SENSITIVITY-DRIVEN, i.e., EARLY-TYPE, SAMPLE}

Our objective for this sample is to find stars for which LBTI can make its most sensitive observations of $\sim 300 \mathrm{~K}$ dust, regardless of the spectral type of the host star. To create this sample, we select all stars that have $N$-band flux densities $\geqslant 1 \mathrm{Jy}$ 
Table 2

Stars in the Sensitivity-driven Sample

\begin{tabular}{|c|c|c|c|c|c|c|c|c|c|}
\hline HD & Name & R.A. & Decl. & $\begin{array}{l}\text { Distance } \\
\quad(\mathrm{pc})\end{array}$ & $\begin{array}{l}\text { Spectral } \\
\text { Type }\end{array}$ & EEID & $\begin{array}{c}F_{v}(N \text { band }) \\
(\mathrm{Jy})\end{array}$ & $\begin{array}{c}\text { Hot/Warm } \\
\text { Excess }\end{array}$ & $\begin{array}{c}\text { Cold } \\
\text { Excess }\end{array}$ \\
\hline HD 33111 & bet Eri & 05:07:51.0 & $-05: 05: 11.2$ & 27.4 & A3IV & 0.248 & 3.72 & n (E14) & y (G13) \\
\hline HD 38678 & zet Lep & $05: 46: 57.3$ & $-14: 49: 19.0$ & 21.6 & A2IV-V & 0.176 & 2.06 & y (FA98), n (A13) & y (FA98) \\
\hline HD 40136 & eta Lep & $05: 56: 24.3$ & $-14: 10: 03.7$ & 14.9 & F2V & 0.161 & 2.36 & & y (L09) \\
\hline HD 81937 & h UMa & 093131.7 & $+63: 03: 42.8$ & 23.8 & FOIV & 0.168 & 2.55 & & n (B06) \\
\hline HD 95418 & beta UMa & $11: 01: 50.5$ & $+56: 22: 56.7$ & 24.4 & A1IV & 0.316 & 4.20 & y (FA98), n (A13) & y (S06) \\
\hline HD 97603 & del Leo & $11: 14: 06.5$ & $+20: 31: 25.4$ & 17.9 & A5IV & 0.278 & 3.90 & $\mathrm{n}(\mathrm{A} 13)$ & n (G13) \\
\hline HD 102647 & bet Leo & 11:49:03.6 & $+14: 34: 19.4$ & 11.0 & $\mathrm{~A} 3 \mathrm{~V}$ & 0.336 & 6.85 & y (A13) & y (S06) \\
\hline HD 103287 & gam Uma & $11: 53: 49.8$ & $+53: 41: 41.1$ & 25.5 & A1IV & 0.308 & 3.69 & & n (S06) \\
\hline HD 105452 & alf Crv & $12: 08: 24.8$ & $-24: 43: 44.0$ & 14.9 & F1V & 0.139 & 1.97 & & n (G13) \\
\hline HD 106591 & del UMa & $12: 15: 25.6$ & $+57: 01: 57.4$ & 24.7 & A2V & 0.199 & 2.00 & n (A13) & n (G13) \\
\hline HD 108767 & del Crv & $12: 29: 51.8$ & $-16: 30: 55.6$ & 26.6 & A0IV & 0.251 & 2.25 & y (E14) & n (S06) \\
\hline HD 109085 & eta Crv & $12: 32: 04.2$ & $-16: 11: 45.6$ & 18.3 & $\mathrm{~F} 2 \mathrm{~V}$ & 0.125 & 1.76 & n (A13) & y (W05) \\
\hline HD 128167 & sig Boo & $14: 34: 40.8$ & $+29: 44: 42.4$ & 15.8 & $\mathrm{~F} 2 \mathrm{~V}$ & 0.117 & 1.39 & & y (L02) \\
\hline HD 129502 & $107 \mathrm{Vir}$ & $14: 43: 03.6$ & $-05: 39: 29.5$ & 18.3 & $\mathrm{~F} 2 \mathrm{~V}$ & 0.151 & 2.60 & n (E14) & n (G13) \\
\hline HD 164259 & zet Ser & 18:00:29.0 & $-03: 41: 25.0$ & 23.6 & F2IV & 0.106 & 1.14 & n (E14) & n (L09) \\
\hline HD 172167 & Vega & $18: 36: 56.3$ & $+38: 47: 01.3$ & 7.7 & $\mathrm{~A} 0 \mathrm{~V}$ & 0.916 & 38.55 & y (A13) & y (G86) \\
\hline HD 187642 & Altair & $19: 50: 47.0$ & $+08: 52: 06.0$ & 5.1 & A7V & 0.570 & 21.63 & y (A13) & n (R05) \\
\hline HD 203280 & Alderamin & $21: 18: 34.8$ & $+62: 35: 08.1$ & 15.0 & A8V & 0.294 & 7.04 & y (A13) & $\mathrm{n}(\mathrm{C} 05)$ \\
\hline HD 210418 & tet Peg & $22: 10: 12.0$ & $+06: 11: 52.3$ & 28.3 & A1V & 0.179 & 1.61 & n (E14) & n (S06) \\
\hline HD 216956 & Fomalhaut & $22: 57: 39.0$ & $-29: 37: 20.0$ & 7.7 & A4V & 0.504 & 15.41 & y (L13) & y (G86) \\
\hline
\end{tabular}

Notes. Excess References: A13 = Absil et al. (2013); B06 = Bryden et al. (2006); C05 = Chen et al. (2005); E14 = Ertel et al. (2014); FA98 = Fajardo-Acosta et al. (1998); G13 = Gáspár et al. (2013); G86 = Gillett (1986); L02 = Laureijs et al. (2002); L09 = Lawler et al. (2009); L13 = Lebreton et al. (2013); R05 = Rieke et al. (2005); S06 = Su et al. (2006); W05 = Wyatt et al. (2005).

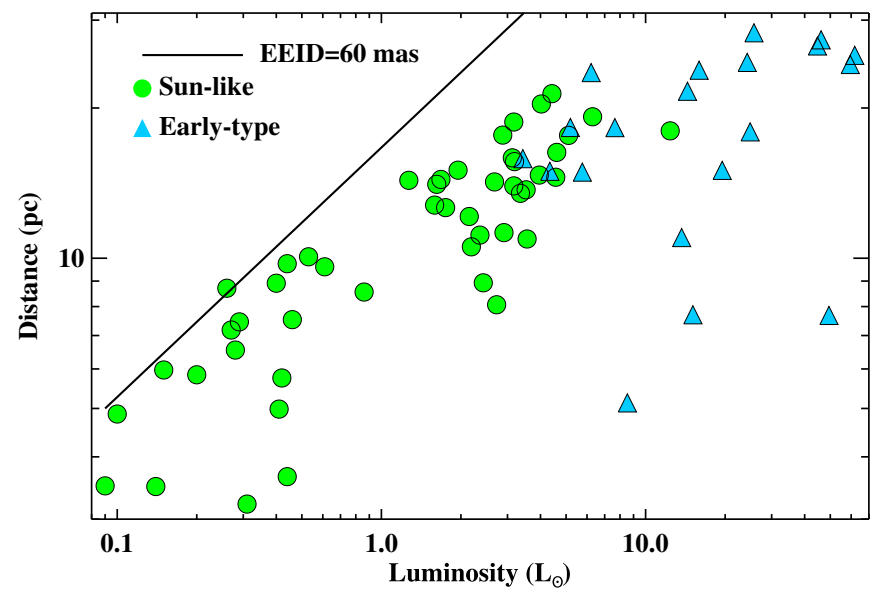

Figure 2. Distance vs. luminosity for the complete sample. The black line shows an Earth equivalent insolation distance (EEID) of 60 mas. Stars that fall above this line were excluded because their exozodi would largely fit within the first null, and therefore LBTI would not be very sensitive to such dust. The LBTI the inner working angle in the $N$ band $(11 \mu \mathrm{m})$, defined as $\lambda / 4 \mathrm{~B}$, is $\approx 39$ mas for the LBT mirror separation of $14.4 \mathrm{~m}$ while the first transmission peak is at 79 mas. That stars $>1 L_{\odot}$ fall well below the line shows that the $N$-band flux density requirement drives the source selection rather than the EEID requirement.

and for which the location of the EEID is $>60$ mas. This preferentially selects A-type and early F-type stars. In general, these are not good exoEarth imaging targets themselves, because of the low habitable-zone-planet-to-star contrast. However, they will provide an important addition to our understanding of the exozodiacal dust luminosity function as it might depend on mass and luminosity of the host star.

We find an additional 20 stars that meet our selection criteria and were not already selected in the Sun-like Sample. These stars are all earlier spectral type than F4 and are given in Table 2. These stars are typically further away than the Sun-like samples tars, with an average distance of $18.6 \mathrm{pc}$. Twelve stars have significant infrared excesses indicating abundant circumstellar dust at some distance from the stars; references are given in the table.

\section{DISCUSSION}

Despite our attempts to reject known binaries (see Section 2.2), there could be unknown companions that would transmit through the LBTI null pattern and therefore generate some signal. There are some ways to distinguish a companion from a disk using LBTI. A companion will transmit primarily through a single LBTI fringe, unlike a spatially resolved disk. Therefore, a companion will produce a null that varies as the orientation of the projected baseline of the interferometer changes due to Earth's rotation over an observation. However, an inclined disk would have a similar effect; therefore, distinguishing a companion from a disk will likely require follow-up observations. For example, measuring the source null in narrower filters at the short and long wavelength ends of the $N$ band, i.e., 8 and 12.5 $\mu \mathrm{m}$, would provide some information on its temperature and spatial extent. Radial velocity observations will constrain the possible masses and orbital periods of suspected companions.

Any companions discovered by LBTI are likely to be of substellar mass. All but four of the Sun-like sample stars and seven of the Early-type sample stars have been studied extensively by radial velocity planet search programs (e.g., Butler et al. 2006; Lagrange et al. 2009; Fischer et al. 2014). At the separation of maximum transmission, i.e., 79 mas, a $80 M_{\text {Jup }}$ brown dwarf in an orbit inclined at $45^{\circ}$ would induce a typical reflex motion of about $2 \mathrm{~km} \mathrm{~s}^{-1}$ for our sample stars, which could be detected for all but the most rapidly rotating stars in the sample.

In advance of scheduled HOSTS observing runs, a prioritized list of targets will be constructed based on the target observability (e.g., above airmass 1.5 for more than $2 \mathrm{hr}$ ) and our expected sensitivity to exozodi. To determine our expected sensitivity, we pass an exozodi model, described in Kennedy et al. (2014), 


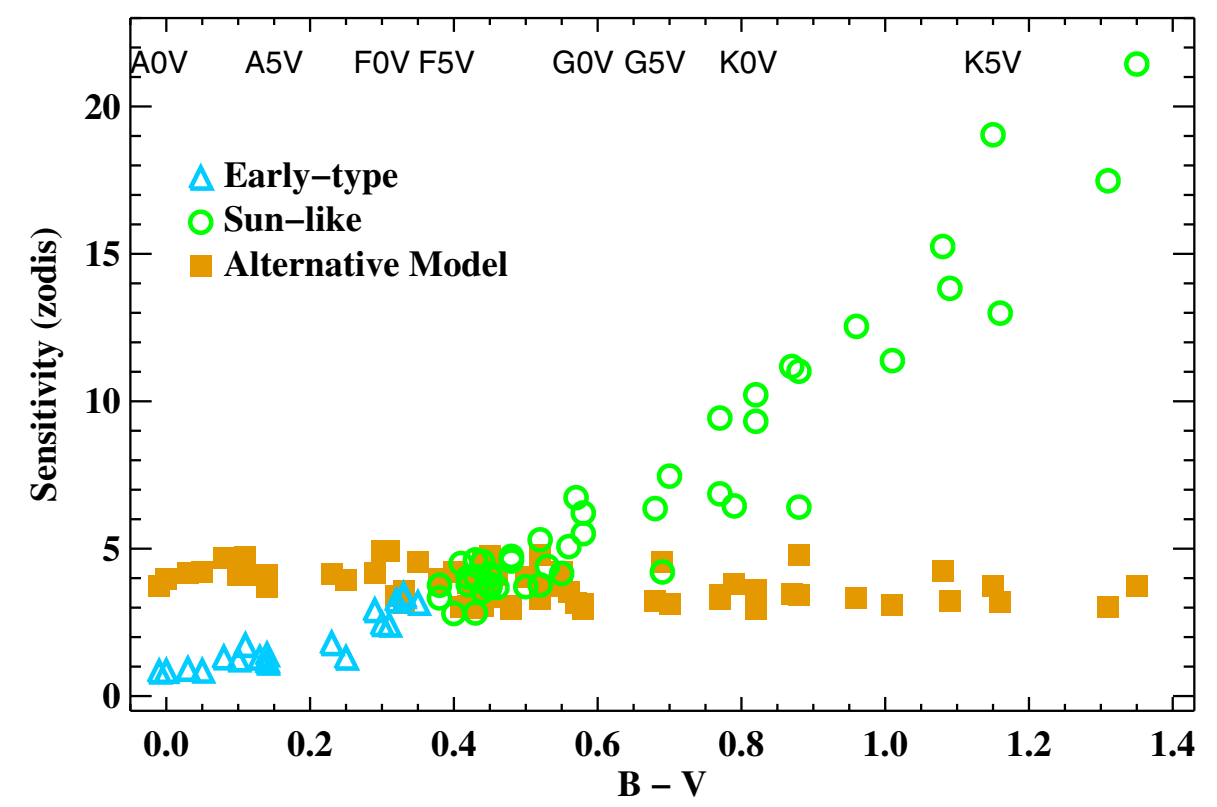

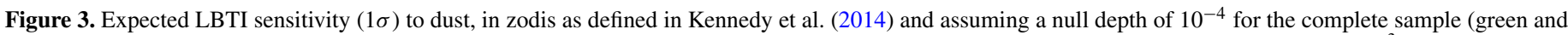

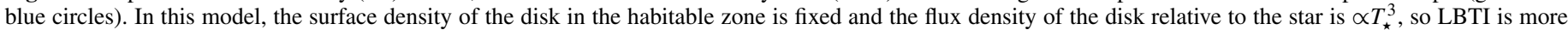

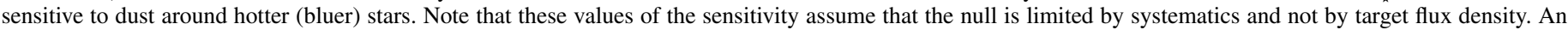

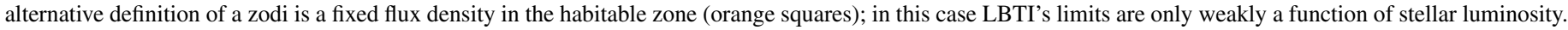

through an LBTI null model to calculate an exozodi limit for each star, in the unit of a "zodi." This model was designed to be simple, to facilitate comparisons between observations of stars of varying properties, and to have a straightforward correspondence with the solar system's actual Zodiacal cloud. The basic features of this model are a fixed surface density in the habitable zone, i.e., at a temperature of $278 \mathrm{~K}$, and a weak power-law dependence of surface density on radius from the star that matches the Zodiacal cloud's. Figure 3 shows this estimation based on current knowledge of the achievable LBTI null depth. LBTI is still in commissioning, so the final dependence of null depth on target brightness is not yet well established. We have assumed that for targets brighter than $2 \mathrm{Jy}$, LBTI will be systematics limited, so the target's flux density will not affect our zodi sensitivity. However, there may be additional degradation of the null for targets of 1-2 Jy, which comprise 28/68 of our targets.

There are many other possible definitions of a "zodi" including ones defined in terms of a fixed $F_{\text {dust }} / F_{\star}$ or $L_{\text {dust }} / L_{\star}$ at a given temperature (Roberge et al. 2012). For comparison purposes, we also calculate an exozodi sensitivity for each of our target stars by assuming a version of our reference model that contains dust only in the habitable zone, i.e., extending from $0.95-1.37 \sqrt{L_{*}}$ and normalized to a fixed $F_{\text {dust }} / F_{\star}=5 \times 10^{-5}$ at a wavelength of $11 \mu \mathrm{m}$ (for our NOMIC filter). These limits are also shown in Figure 3.

The goal of the overall survey is not only to identify exozodiacal dust around specific stars of interest, but also to measure the luminosity function of disks in a general statistical sense. As such, we define here a key metric for the overall survey $-Z 10$ - the fraction of stars with more than 10 zodis. This level of exozodi, versus a solar system level of dust, would cut the number of Earth-like planets imaged by a future directimaging mission by half (Stark et al. 2014). This recent work also shows, however, that a mission that observes an ensemble of stars has a total planet yield that is a weak function of the exozodi level (Stark et al. 2014).
In a real-world ground-based observing program, under changing seeing and transparency and seasonally biased conditions, it will be impossible to observe all stars, even those brighter than $2 \mathrm{Jy}$, to identical zodi detection depths. Of the 68 stars in Tables 1 and 2 , we expect to observe $\sim 50$. What is critical is that no biases to the sample are introduced during the selection of the actual observed targets.

The ability of the LBTI survey to constrain Z10 depends on both the number of observed targets and the sensitivity of each individual measurement. We performed Monte Carlo simulations to estimate the expected accuracy of $Z 10$ as a function of the number of targets. Assuming that the underlying distribution of disk brightnesses follows a log-normal distribution whose width is set by $Z 10$, we determine how well $Z 10$ is constrained by the LBTI observations. We assume that each star is treated as a unique observation. The bright end of the distribution is already constrained by Spitzer/KIN/WISE observations (Lawler et al. 2009; Millan-Gabet et al. 2011; Kennedy \& Wyatt 2013); therefore, we set the frequency of 1000 zodi disks to be $1 \%$.

At first we consider a uniform survey depth with 3 zodi sensitivity for each measurement $(1-\sigma)$, which we assume would be the perfect, likely unachievable, survey LBTI could perform. Figure 4 shows how well $Z 10$ is constrained by uniformdepth surveys ranging from 30 to 70 stars. We find that a 50 star survey can measure $Z 10$ with $\sim 20 \%$ accuracy (for $Z 10 \simeq 0.3-0.4)$. Using advanced statistical methods to allow averaging over multiple targets to achieve deeper zodi limits, it may be possible to improve on these rough limits (Mennesson et al. 2014).

Since variations in weather will inevitably result in nonuniform sensitivity, Figure 4 also shows the constraints on Z10 for a two-layered survey, where 40 stars are observed with 3 zodi accuracy and another 30 stars with only 10 zodi accuracy. We find that this layered survey has equivalent power to reveal the zodi luminosity function as a 50 star survey done to a depth of 3 zodis. We conclude that an optimal observing strategy should 
Table 3

Binary Stars Excluded from the Sample

\begin{tabular}{|c|c|c|c|}
\hline HD & Name & SpTyp & Binarity Notes \\
\hline HD 432 & bet Cas & F2IV & WDS says SB with $P=27 \mathrm{~d}$ \\
\hline HD 4614 & eta Cas & G3V & VB $12^{\prime \prime}, 70 \mathrm{AU}, P=480 \mathrm{yr}$ \\
\hline HD 6582 & mu Cas & G5V & $\mathrm{VB} 1^{\prime \prime}, 7.5 \mathrm{AU}+\mathrm{SB}$ \\
\hline HD 8538 & del Cas & A5III-IV & SB, perhaps eclipsing \\
\hline HD 11443 & alf Tri & F6IV & $\mathrm{SB}, P=1.8 \mathrm{~d}$ \\
\hline HD 11636 & bet Ari & $\mathrm{A} 5 \mathrm{~V}$ & $\mathrm{SB} 9, P=107 \mathrm{~d}$ \\
\hline HD 13161 & & A5IV & $\mathrm{SB}, P=31 \mathrm{~d}$, resolved by MarkIII \\
\hline HD 13974 & & G0V & $\mathrm{SB}, P=10 \mathrm{~d}$ \\
\hline HD 16970 & & $\mathrm{~A} 2 \mathrm{~V}$ & $\mathrm{VB}, 2^{\prime \prime} .3$ \\
\hline HD 20010 & & F6V & VB 4..4, $62 \mathrm{AU}$ \\
\hline HD 20630 & kap01 Cet & G5V & WDS says SB, but not variable in Nidever et al. (2002) \\
\hline HD 39587 & chi1 Ori & G0V & VB $0.7,6 \mathrm{AU}, P=14 \mathrm{yr}$ \\
\hline HD 40183 & & A1IV-V & $\mathrm{SB}, P=3.7 \mathrm{~d}$, resolved by MarkIII \\
\hline HD 47105 & & A1.5IV & SB/speckle VB \\
\hline HD 48915 & Sirius & $\mathrm{A} 0 \mathrm{~V}$ & VB, $7^{\prime \prime} .5$ \\
\hline HD 56986 & del Gem & F2V & $\mathrm{SB}, P=6.1 \mathrm{yr}$ \\
\hline HD 60179 & Castor A & A1.5IV & $\mathrm{SB}, P=9.2 \mathrm{~d}$ plus $\mathrm{VB}, P=467 \mathrm{yr}$ \\
\hline HD 61421 & Procyon & F5IV & VB $5^{\prime \prime}, 18 \mathrm{AU}, P=40 \mathrm{yr}$ and SB \\
\hline HD 76644 & & $\mathrm{~A} 7 \mathrm{~V}$ & $\mathrm{SB}, P=11 \mathrm{yr}$ \\
\hline HD 76943 & $10 \mathrm{UMa}$ & F3V & SB/VB $0 . ' 6, P=21.8$ yr \\
\hline HD 82328 & tet UMa & F7V & WDS says SB (SBC7), but not in SB9, also VB, $5^{\prime \prime}$ \\
\hline HD 82885 & SV Lmi & G8III & VB $3 . \prime 8,43 \mathrm{AU}, P=200 \mathrm{yr}$ \\
\hline HD 95735 & & $\mathrm{M} 2 \mathrm{~V}$ & $\mathrm{~EB}$ \\
\hline HD 98231 & GJ 423A & G0V & Complicated multiple system \\
\hline HD 104304 & & G8IV & $\mathrm{VB}, 1^{\prime \prime}$ \\
\hline HD 109358 & bet Cvn & G0V & $\mathrm{SB}, 0 . \prime 1,1.3 \mathrm{AU}$ \\
\hline HD 110379 & GJ 482A & F0V & $\mathrm{VB}, 3^{\prime \prime} .75,44 \mathrm{AU}, P=171 \mathrm{yr}$ \\
\hline HD 112413 & alf02 CVn & A0II-III & SB \\
\hline HD $114378 J$ & alph Com & F5V & VB $0.7,12 \mathrm{AU}, P=26 \mathrm{yr}$ \\
\hline HD 114710 & bet Com & G0V & possible SB \\
\hline HD 116656 & Mizar A & $\mathrm{A} 1.5 \mathrm{~V}$ & $\mathrm{SB}, P=20.5 \mathrm{~d}$ \\
\hline HD 118098 & zet Vir & A3V & $\mathrm{VB}$, companion is M4-M7 \\
\hline HD 121370 & eta Boo & G8V & $\mathrm{SB} 9, P=494 \mathrm{~d}$ \\
\hline HD 130841 & alf Lib A & A4IV-V & possible SB \\
\hline HD 131156 & 37 Boo & G8V & VB $4^{\prime \prime} .9,33 \mathrm{AU}, P=151 \mathrm{yr}$ \\
\hline HD 131511 & GJ 567 & $\mathrm{~K} 2 \mathrm{~V}$ & possible $\mathrm{SB} P=125 \mathrm{~d}$ \\
\hline HD 133640 & GJ 575 & G0V & VB, 3..8, 48 AU (now at 1..5) \\
\hline HD 139006 & alf $\mathrm{CrB}$ & A1IV & $\mathrm{SB}, P=17 \mathrm{~d}$ \\
\hline HD 140538 & & $\mathrm{G} 2.5 \mathrm{~V}$ & $\mathrm{VB}, 4^{\prime \prime} .4,65 \mathrm{AU}$ \\
\hline HD 144284 & tet Dra & F8 IV-V & $\mathrm{SB}, P=3.1 \mathrm{~d}$ \\
\hline HD 155125 & & A2IV-V & VB $0 . .86$ \\
\hline HD 155886 & GJ 663A & $\mathrm{K} 2 \mathrm{~V}$ & VB $5^{\prime \prime}, 28 \mathrm{AU}$ \\
\hline HD 155885 & $36 \mathrm{Oph}$ & $\mathrm{K} 2 \mathrm{~V}$ & VB $15^{\prime \prime}, 87 \mathrm{AU}, P=569 \mathrm{yr}+$ possible SB \\
\hline HD 156164 & del Her & A1IV & SB 0.1 \\
\hline HD 156897 & $40 \mathrm{Oph}$ & $\mathrm{F} 2 \mathrm{~V}$ & VB, approx $4^{\prime \prime}$ (CCDM) \\
\hline HD 159561 & alf Oph & A5II & VB 0.18 \\
\hline HD 160269 & & G0V & VB $1^{\prime \prime} .5,21 \mathrm{AU}$ \\
\hline HD 160346 & GJ 688 & $\mathrm{~K} 3 \mathrm{~V}$ & SB $P=84 \mathrm{~d}$ \\
\hline HD 161797 & mu Her & G5IV & VB 1".4, 12AU, $P=65 \mathrm{yr}$ \\
\hline HD 165341 & 70 Oph A & $\mathrm{K} 0 \mathrm{~V}$ & VB 4.'6, 23AU, $P=88 \mathrm{yr}$ \\
\hline HD 165908 & b Her & F7V & VB $1^{\prime \prime} .1,17 \mathrm{AU}, P=26 \mathrm{yr}$ \\
\hline HD 170153 & & F7V & $\mathrm{SB} 0^{\prime \prime} 1,1 \mathrm{AU}, P=0.8 \mathrm{yr}$ \\
\hline CCDM 19026-2953 A & & $\mathrm{A} 2.5 \mathrm{~V}$ & VB $0 . .53$ \\
\hline HD 177724 & & A0IV-V & $\mathrm{SB}+\mathrm{VB}, 5^{\prime \prime}$ \\
\hline HD 182640 & del Aql & F1IV-V & $\mathrm{SB}$, resolved at $0^{\prime \prime} 1$ \\
\hline HD 185395 & tet Cyg & F4V & VB/SB, approx 2..5 \\
\hline HD 207098 & del Cap & F2III & $\mathrm{EB}$ \\
\hline HD 210027 & iot Peg & F5V & $\mathrm{SB} 1, P=10 \mathrm{~d}$ \\
\hline HD 224930 & 85 Peg & G5V & VB $0^{\prime \prime} 8,10 \mathrm{AU}, P=26 \mathrm{yr}$ \\
\hline
\end{tabular}

not mandate uniform sensitivity, thereby concentrating a large fraction of telescope time on a small number of stars, but will instead observe a greater number of stars, some with greater depth than others.
The HOSTS survey is expected to begin in 2015 and to continue for two to three years. During commissioning, LBTI observed $\eta \mathrm{Crv}$, one of the early-type sample stars with a known mid-infrared excess. The observations demonstrate the power 


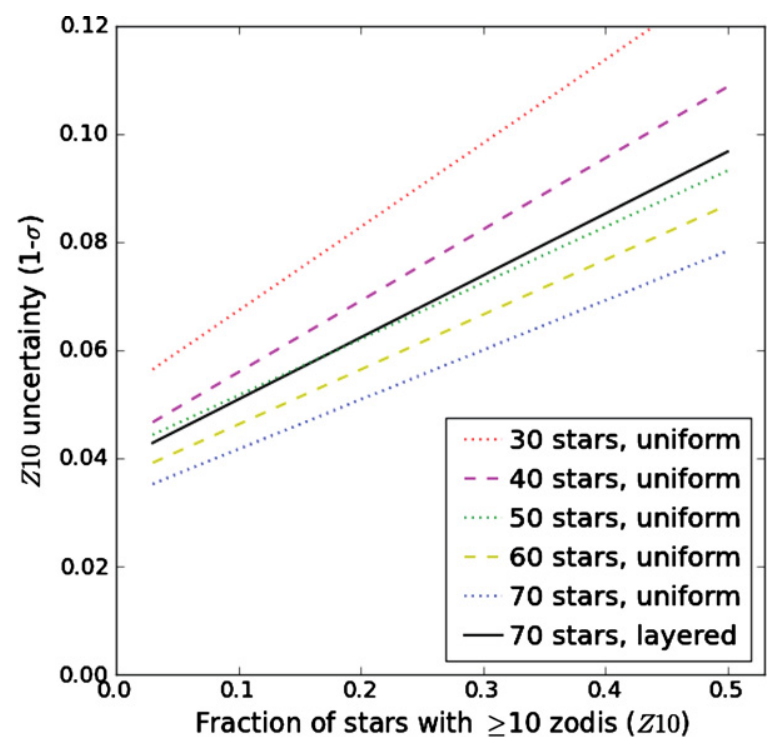

Figure 4. Ability of the overall survey to constrain $Z 10$ (the fraction of stars with $\geqslant 10$ zodis of dust) depends on the number of targets and the accuracy of each measurement. Based on Monte Carlo simulations we find that a survey with two levels of sensitivity -40 stars with 3 zodi accuracy and 30 stars with 10 zodi accuracy (black line) - is roughly equivalent to a 50 star survey of uniform 3 zodi depth (green dashed line).

of LBTI to constrain the dust distribution in the habitable zone (Defrere et al. 2014).

The Large Binocular Telescope Interferometer is funded by the National Aeronautics and Space Administration as part of its Exoplanet Exploration Program. This work of G.M.K. and M.C.W. was supported by the European Union through ERC grant No. 279973. This research has made use of the SIMBAD database and the VizieR catalog access tool, CDS, Strasbourg, France and the Washington Double Star Catalog maintained at the U.S. Naval Observatory.

\section{APPENDIX}

\section{BINARY STARS EXCLUDED FROM SAMPLE}

Here, we list stars that could otherwise meet our selection criteria but were excluded due to binarity as described in Section 2.2. Much of the information in Table 3 comes from Washington Double Star Catalog (Mason et al. 2013).

\section{REFERENCES}

Absil, O., Defrère, D., du Foresto, V. C., et al. 2013, A\&A, A104 Andrews, S. M., \& Williams, J. P. 2005, ApJ, 631, 1134

Beichman, C. A., Bryden, G., Stapelfeldt, K. R., et al. 2006, ApJ, 652, 1674
Bryden, G., Beichman, C. A., Carpenter, J. M., et al. 2009, ApJ, 705, 1226 Bryden, G., Beichman, C. A., Trilling, D. E., et al. 2006, ApJ, 636, 1098 Butler, R. P., Wright, J. T., Marcy, G. W., et al. 2006, ApJ, 646, 505

Chen, C. H., Mittal, T., Kuchner, M., et al. 2014, ApJS, 211, 25

Chen, C. H., Patten, B. M., Werner, M. W., et al. 2005, ApJ, 634, 1372

Christiansen, J. L., Jenkins, J. M., Caldwell, D. A., et al. 2012, PASP, 124, 1279

Defrère, D., Absil, O., den Hartog, R., Hanot, C., \& Stark, C. 2010, A\&A, 509, A9

Defrère, D., Hinz, P., Downey, E., et al. 2014, Proc. SPIE, 9146, 914609

Defrere, D., Hinz, P. M., Sekmer, A. J., et al. 2014, ApJ, in press

Drilling, J. S., \& Landolt, A. U. 2000, in Allen's Astrophysical Quantities, ed. A. N. Cox (New York: AIP), 381

Eiroa, C., Marshall, J. P., Mora, A., et al. 2013, A\&A, 555, 11

Ertel, S., Absil, O., Defrère, D., et al. 2014, A\&A, 570, A128

Fajardo-Acosta, S. B., Telesco, C. M., \& Knacke, R. F. 1998, AJ, 115, 2101

Fischer, D. A., Marcy, G. W., \& Spronck, J. F. P. 2014, ApJS, 210, 5

Gáspár, A., Rieke, G. H., \& Balog, Z. 2013, ApJ, 768, 25

Gillett, F. C. 1986, in Light on Dark Matter, ed. F. P. Israel (Astrophysics and Space Science Library, Vol. 124; Dordrecht: Reidel), 61

Greaves, J. S., Holland, W. S., Jayawardhana, R., Wyatt, M. C., \& Dent, W. R. F. 2004, MNRAS, 348, 1097

Greaves, J. S., \& Wyatt, M. C. 2003, MNRAS, 345, 1212

Greene, T., Noecker, C., \& ExoPAG SAG 5 team. 2013, arXiv:1303.6707

Harris, R. J., Andrews, S. M., Wilner, D. J., \& Kraus, A. L. 2012, ApJ, 751,115

Hinz, P., Arbo, P., Bailey, V., et al. 2012, Proc. SPIE, 8445, 0

Hinz, P. M., Solheid, E., Durney, O., \& Hoffmann, W. F. 2008, Proc. SPIE, 7013, 39

Jensen, E. L. N., Mathieu, R. D., \& Fuller, G. A. 1996, ApJ, 458, 312

Kennedy, G. M., \& Wyatt, M. C. 2013, MNRAS, 433, 2334

Kennedy, G. M., Wyatt, M. C., Bailey, V., et al. 2014, ApJS, 216, 23

Koerner, D. W., Kim, S., Trilling, D. E., et al. 2010, ApJ, 710, L26

Kopparapu, R. K., Ramirez, R., Kasting, J. F., et al. 2013, ApJ, 765, 131

Lagrange, A.-M., Desort, M., Galland, F., Udry, S., \& Mayor, M. 2009, A\&A, 495,335

Laureijs, R. J., Jourdain de Muizon, M., Leech, K., et al. 2002, A\&A, 387,285

Lawler, S. M., Beichman, C. A., Bryden, G., et al. 2009, ApJ, 705, 89

Lebreton, J., van Lieshout, R., Augereau, J.-C., et al. 2013, A\&A, 555, A146

Leconte, J., Forget, F., Charnay, B., Wordsworth, R., \& Pottier, A. 2013, Natur, 504,268

Mason, B. D., Wycoff, G. L., Hartkopf, W. I., Douglass, G. G., \& Worley, C. E. 2013, yCat, 1, 2026

Mennesson, B., Millan-Gabet, R., Serabyn, E., et al. 2014, ApJ, 797, 119

Millan-Gabet, R., Serabyn, E., Mennesson, B., et al. 2011, ApJ, 734, 67

Nidever, D. L., Marcy, G. W., Butler, R. P., Fischer, D. A., \& Vogt, S. S. 2002, ApJS, 141, 503

Osterloh, M., \& Beckwith, S. V. W. 1995, ApJ, 439, 288

Phillips, N. M., Greaves, J. S., Dent, W. R. F., et al. 2010, MNRAS, 403, 1089

Pourbaix, D., Tokovinin, A. A., Batten, A. H., et al. 2009, yCat, 1, 2020

Rieke, G. H., Su, K. Y. L., Stansberry, J. A., et al. 2005, ApJ, 620,1010

Roberge, A., Chen, C. H., Millan-Gabet, R., et al. 2012, PASP, 124, 799 Spergel, D., Gehrels, N., Breckinridge, J., et al. 2013, arXiv:1305.5422

Stark, C. C., Roberge, A., Mandell, A., \& Robinson, T. D. 2014, ApJ, 795, 122 Su, K. Y. L., Rieke, G. H., Stansberry, J. A., et al. 2006, ApJ, 653, 675

Trilling, D. E., Bryden, G., Beichman, C. A., et al. 2008, ApJ, 674, 1086

Turnbull, M. C., Glassman, T., Roberge, A., et al. 2012, PASP, 124, 418

Wyatt, M. C., Greaves, J. S., Dent, W. R. F., \& Coulson, I. M. 2005, ApJ, 620,492

Wyatt, M. C., Smith, R., Greaves, J. S., et al. 2007, ApJ, 658, 569 